\title{
Schizophrénie et activité physique : EPI or not EPI ?
}

\author{
M. Duclos \\ (C) Lavoisier SAS 2015
}

Les patients souffrant de schizophrénie présentent une surmortalité comparativement à la population générale. Même si la première cause de cette augmentation reste le suicide, ils présentent aussi une surmortalité cardiovasculaire par coexistence de plusieurs facteurs de risques cardio-métaboliques (obésité, diabète de type 2 , dyslipidémies, hypertension artérielle). L'incidence élevée de l'obésité et des maladies cardiovasculaires chez les schizophrènes est d'origine multifactorielle : génétique, nutritionnelle (alimentation déséquilibrée, effets des traitements antipsychotiques), conditions socio-économiques défavorables (faibles ressources financières et isolement) et mode de vie inactif (faible niveau d'activité physique). Le manque d'activité physique chez les patients schizophrènes peut être lié à l'action sédative des traitements médicamenteux, mais aussi à la symptomatologie négative. Cette dernière, marquée par une diminution des capacités fonctionnelles des sujets, regroupe l'émoussement affectif, la perte de volonté, l'anhédonie, l'apathie, le repli sur soi, le retrait affectif, la baisse des intérêts. Ces symptômes parfois difficiles à repérer, seraient présents chez 50 à $90 \%$ des patients en début de maladie [1] et persistent après traitement chez 35 à $70 \%$ des patients [2]. A long terme, ces symptômes négatifs ont un impact sur la sédentarité et le niveau d'activité physique des patients aggravant le risque de surpoids et d'obésité mais aussi de diabète de type 2 et de pathologies cardiovasculaires.

Plusieurs études rapportent un effet bénéfique d'un programme structuré et contrôlé d'activité physique sur les paramètres reflétant l'état de santé cardio-métabolique mais aussi sur la symptomatologie psychotique des patients schizophrènes [3].

L'activité physique fait partie de la prise en charge à part entière des sujets à risques cardio-métaboliques élevés et du diabète de type 2. Dans ces conditions, il ne semblerait pas déraisonnable de proposer que l'activité physique puisse faire partie de la prise en charge de patients souffrant de schizophrénie.

M. Duclos $(\square)$

Service de Médecine du Sport et des Explorations Fonctionnelles, CHU Gabriel Montpied ; Laboratoire de Nutrition Humaine,

INRA UMR 1019, Université d'Auvergne I,

63000 Clermont-Ferrand

e-mail : mduclos@chu-clermontferrand.fr
Pour répondre à cette proposition qui se justifie d'autant plus dans le contexte actuel d'une médecine orientée vers la prévention, au moins la prévention primaire des sujets à risques cardio-métaboliques élevés, il faut prouver qu'un programme d'activité physique structuré est faisable (pas d'effet secondaire et bonne adhésion), reproductible et conduit à une diminution significative des risques cardio-métaboliques chez des sujets schizophrènes.

La revue de Chalfoun et al répond positivement à la question de l'efficacité sur les risques cardio-métaboliques mais avec une proposition d'activité physique qui peut surprendre au premier abord : l'entraînement par intervalles à haute intensité (EPI). Qu'est-ce que l'EPI ? C'est une activité physique reposant sur des périodes d'exercice aérobie de haute intensité (ex. pédalage 30 secondes sur ergocycle à une fréquence cardiaque égale à $90 \%$ de la fréquence cardiaque maximale) entrecoupées par des périodes de récupération d'intensité basse à modérée ( 90 secondes à $50 \%$ de la fréquence cardiaque maximale). L'intérêt repose sur sa durée courte (30 minutes tout compris intégrant les temps d'échauffement et de récupération), remplaçant les longues séances monotones de 30 à 45 minutes de l'exercice modéré continu, sur sa fréquence diminuée à deux fois par semaine du fait de l'intensité (au lieu de 3 à 5 fois par semaine), ce qui favorise le maintien à long terme de l'activité physique, élément primordial même si on manque encore de ce recul à long terme chez les patients schizophrènes. Enoncé à brûle pourpoint cela peut paraître peu réaliste voire dangereux, pourtant ce type d'activité physique a fait la preuve de sa faisabilité et de son efficacité sur les paramètres cardio-métaboliques en population générale mais aussi chez des sujets porteur de pathologies chroniques : obésité, diabète de type 2 , insuffisance cardiaque et schizophrénie.

La revue de Chalfoun et al pose les bonnes questions, s'accompagne de conseils et suggestions pour lever les barrières (des soignants) face à la mise en place, la pratique et la faisabilité de cette activité physique. Elle ouvre de nouvelles perspectives d'accompagnement des patients schizophrènes.

EPI or not EPI ? Telle n'est pas la question. Ce serait plutôt : mais qu'est-ce qu'on attend pour faire bouger les patients atteints de schizophrénie? 


\section{Références}

1. Bottlender R, Sato T, Jager M, et al (2003) Does considering duration of negative symptoms increase their specificity for schizophrenia? Schizophr Res 60:321-2
2. Malla AK, Norman RM, McLean TS, et al (2004) Determinants of quality of life in first-episode psychosis. Acta Psychiatr Scand 109:46-54

3. Gorczynski P, Faulkner G (2010) Exercise therapy for schizophrenia. Schizophr Bull 36:665-6 